\title{
Block Window Retransmission ARQ Scheme for Next Generation High Speed IrDA Links
}

\author{
Shawkat Shamim Ara, Alam Mohammad Shah and Mitsuji Matsumoto \\ Global Information and Telecommunication Studies, Waseda University, \\ Tokyo, Japan
}

\begin{abstract}
Indoor infrared links are anticipated to reach $100 \mathrm{Mbit} / \mathrm{s}$ or even higher data rates in the future. At this high data rate, the existing Go-Back- $N$ automatic repeat request (ARQ) scheme requires window and frame size adaptation to the corresponding optimum values for the correspondent Bit Error Rate (BER). But adaptive approaches always add a significant amount of complexity to the system. The aim of this paper is to present a new Automatic Repeat Request (ARQ) scheme, Block Window Retransmission (BWR) ARQ scheme, for achieving higher throughput efficiency over a wide range of BERs without adaptation of parameters. Simulation results show that it significantly outperforms the existing Go-Back- $N$ ARQ scheme for next generation high speed IrDA links. We also examine the effect of window size, frame data length and minimum turn around time on the performance of the BWR ARQ scheme.
\end{abstract}

\section{INTRODUCTION}

The data communication environment has been changing dramatically in the recent years. The wired access network is being changed to an IP-based network from a telephone-based network to support data rate over $100 \mathrm{Mbps}$ whereas the current wireless services still offer data rates much less than $100 \mathrm{Mbit} / \mathrm{s}$. Furthermore, as the trend of using larger files combined with the need for faster file transfer times continues to become more important, there will be a great need for much faster short range wireless links [1]. In contrast, significant advantages have motivated recent interest into infrared wireless communication as a medium for point to point short range indoor communication [2]. However, the infrared (IR) systems also suffer from severe noise and disturbances. Therefore, automatic repeat request (ARQ) error control schemes are used for providing reliable data communications. Infrared Data Association (IrDA) has adopted go-back- $N$ ARQ scheme at infrared link access protocol (IrLAP) [3] layer for its inherent simplicity.

However, the throughput of the existing go-back- $N$ ARQ scheme decreases very sharply with bit error rate (BER) increase at high speed $[4,5]$. Only a few works have been done to investigate the IrLAP for the future high speed infrared links. In [6], Boucouvalas and Vitsas have examined existing IrLAP protocol operating at $100 \mathrm{Mbit} / \mathrm{s}$ and concluded that it offers excellent performance even at $100 \mathrm{Mbit} / \mathrm{s}$, provided IrLAP window size and frame length values are adapted to the corresponding optimum values for the BER [6, 7]. But this adaptive approach adds significant amount of complexity to the system. Hence, in this paper, we present a Block Window
Retransmission (BWR) ARQ scheme for next generation highspeed IrDA links without adapting parameters to the optimum values. We also examine the effect of window size, frame data length and minimum turn around time on the performance of the BWR ARQ scheme. Simulation results show that it significantly outperforms the existing go-back- $N$ ARQ scheme for the future high-speed IrDA links.

\section{EXISTING Go-BACK-N ARQ SCHEME}

In the existing go-back- $N$ (GBN) ARQ scheme on IrDA links, when a received frame is detected in error, the receiver discards that frame and all the subsequent frames of the window regardless of whether or not they are received successfully and sends negative acknowledgment (NACK) to the transmitter for that error frame. Upon receiving a NACK, the transmitter resends the error frame and $N-1$ succeeding frames that were transmitted during the previous window transmission [8]. The inefficiency of this ARQ scheme is caused by the retransmission of many error-free frames following an error frame of a large window.

\section{Proposed Block Window RETRANSMission (BWR) ARQ SCHEME}

The basic difference of our proposed Block Window Retransmission (BWR) ARQ scheme from the existing GoBack- $N$ ARQ scheme is the method of acknowledgement and the retransmission strategy. The key idea of the BWR ARQ scheme is to store all error free but out of sequence frames received at the secondary station following the first erroneous frame in a small buffer and divide the next predicted window in $n$ number of predefined $b$ blocks. If any block within the next window has all frames stored in the buffer of secondary station, it is ignored by the primary station from being retransmitted. The secondary station always informs the primary station about the next expected frame number and a bit map indicating the status of the blocks in its buffer using the existing frame structure. Based on this information, the primary retransmits or transmits only the expected blocks (see Fig. 1).

\section{A. Acknowledgements}

In the BWR scheme, the primary and the secondary station divides all the frames within a window in six predefined $b$ blocks (Fig. 1(c)). The idea behind dividing in six blocks rather than any number is to take the advantage of the existing IrDA 


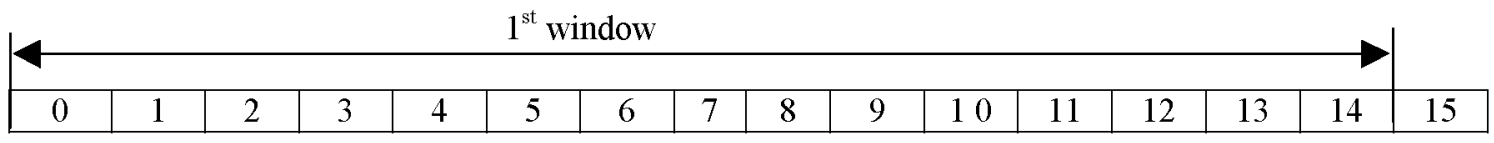

(a)

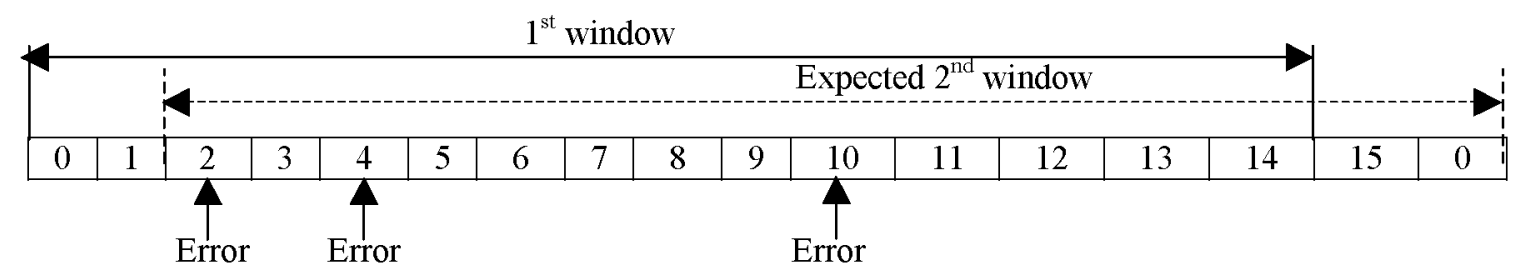

(b)

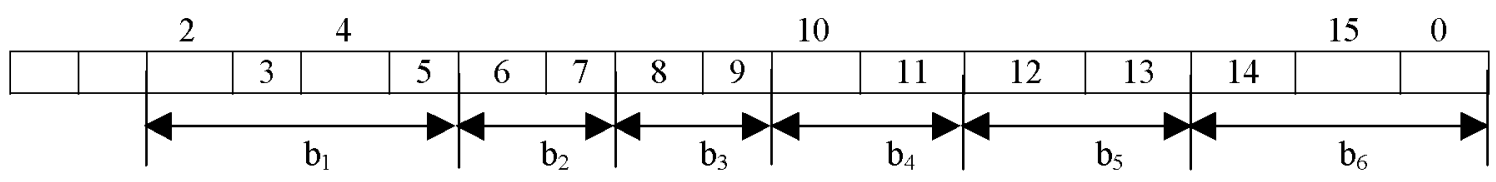

(c)

\begin{tabular}{|l|l|l|l|l|l|l|l|l|l|l|l|l|l|l|l|} 
& $2^{\text {nd }}$ window \\
\hline
\end{tabular}

(d)

Figure 1. (a) Primary station during first window transmission (b) Secondary station during receiving first window (c) Buffer state of the secondary station after first window transmission; Predicted next window is divided in six blocks $b_{1}, b_{2}, b_{3}, b_{4}, b_{5}$ and $b_{6}$. (d) Primary station during second window transmission

proposed extended control field of Supervisory frame $(S$ frame). The receiver assumes that the first and the last blocks always need to be retransmitted or transmitted because at least one frame within these blocks is always not stored in the buffer of the secondary station; unless the first frame in the last window transmission was erroneous. So, the secondary only needs to inform the status of the middle four blocks within the next predicted window. The size of first block $b_{1}$ equals to (window size +1 )/4. The middle four blocks, denoted by $b_{2}, b_{3}$, $b_{4}$ and $b_{5}$, consist of the subsequent (window size +1$) / 8$ frames each. The remaining ((window size +1$) / 4)-1$ frames belong to the last block $b_{6}$.

For our proposed scheme, as we assumed that the secondary has no information data to be transmitted to the primary station, we have only considered the extended control field format of Supervisory frame( $S$-frame). Fig. 2 shows the supervisory frame format for giving acknowledgement. Currently there are four unused bits in the extended control field of $S$-frame. In the proposed BWR ARQ scheme, these four bits are denoted as Block Status (BS) field and contains the bit map information for the middle four blocks (block $b_{2}$, $b_{3}, b_{4}$ and $b_{5}$ ) of the next window. The SS field is used to identify the function of $S$-frame.

\begin{tabular}{|l|l|l|l|l|}
\hline $\operatorname{Nr}(7$ bits $)$ & P/F (1 bit) & Block Status (4 bits) & SS (2 bits) & Frame Type (2 bits) \\
\hline
\end{tabular}

Figure 2. Supervisory frame format for giving acknowledgements

\section{B. Retransmission Strategy}

In the BWR ARQ scheme, the transmission of a large amount of information data from the primary to the secondary station is considered. The secondary does not transmit information to the primary and responds only with Supervisory frame ( $S$-frame). It is also assumed that $S$-frames are small enough to be always received error free. The primary station sends Information frames ( $I$-frame) until the end of the window. In the last frame of the current window, the primary sets the Poll (P) bit to poll the secondary and waits for the acknowledgement as well as the block status information.

At the receiving end, when the secondary station receives a correct in sequence $I$-frame, it passes the frame to the upper layer. It also checks the buffer for the next expected frame whether it is already stored there for being out of sequence in the previous transmissions. If it finds the frame stored there, it also sends that frame to the upper layer to release the corresponding buffer position. The same procedure continues until it can not find the next expected frame in the buffer. But, if the received $I$-frame is not in sequence, the system considers two cases. Either one of the previous $I$-frames in current window transmission was lost or the frame was already stored in the buffer.

For the first case, the system enters into exception state and the frame is stored in the corresponding buffer position. All the subsequent error free frames within the same window are stored in the similar fashion. During the exception state, no 
information data is passed to the upper layer and this state exists until the received frame is the next expected frame. For the other case, the secondary simply discards the frame.

Fig. 1 explains the operation of BWR ARQ scheme for window size $W=15$ frames and the maximum sequence number 15. In Fig. 1(a), the primary station selects the first window from frame number 0 to 14 and sends them accordingly. In Fig. 1(b), the secondary station receives frames error free up to frame sequence number 1 and sends it to the upper layer. But it receives frame 2 erroneously and therefore discards it. When it receives frame 3 correctly but certainly in out of sequence, it enters into the exception state and stores the frame in the corresponding position of buffer. Similarly it stores frame 5, 6 , $7,8,9,11,12,13$ and 14 in the buffer but discards the erroneous frames 4 and 10. In Fig. 1(c), the secondary station predicts the next window starting from frame 2 and divides the whole window in six blocks. It calculates the block status information comparing its buffer. Block b2, b3 and b5 have all frames stored in the buffer. So, the bit map for b2, b3, b4 and b5 equals 1101. Secondary sends this information as well as the next expected frame number which is 2 in this case, to the primary station. In Fig. 1(d), the primary sets the next window from frame 2 to frame 0 and divides it in the same blocks that secondary predicted in advance. Then it sends all the corresponding frames except the frames of blocks b2, b3 and b5.

\section{Performance Analysis}

To examine the performance of the proposed BWR scheme and the effect of different link parameters on it, a set of simulation runs was performed using the $\mathrm{OPNET}^{\mathrm{TM}}$ simulation package [9].

Fig 3 plots throughput efficiency versus BER for the proposed BWR scheme and the existing GBN scheme for $100 \mathrm{Mb} / \mathrm{s}$ link data rate $(C)$ with turn-around time $\left(t_{t a}\right) 0.1 \mathrm{~ms}$ and frame data length $(L) 2 \mathrm{~KB}$. It shows that the BWR ARQ scheme with window size $(W) 127$ frames provides an excellent performance over a wide range of bit error rates for $100 \mathrm{Mbit} / \mathrm{s}$ links. However, in the case of GBN scheme, the employment of large window size 127 results in high throughput $(98 \%)$ for low BER only but renders the link operation very vulnerable to higher BER. But using the proposed BWR scheme with this large window size of 127

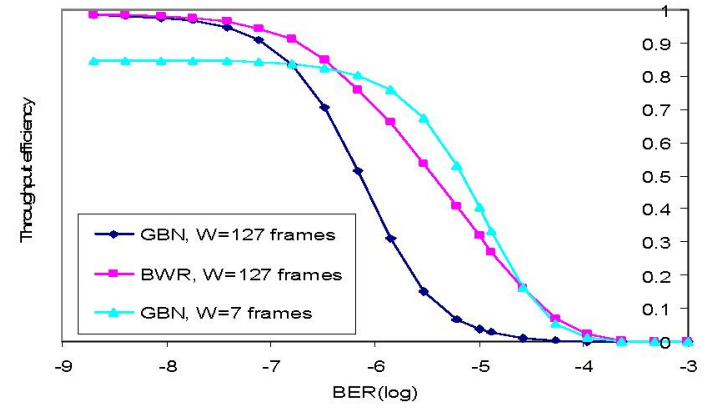

Figure 3. Throughput efficiency versus BER for $t_{t a}=0.1 \mathrm{~ms}, L=2 \mathrm{~KB}$ and $C=100 \mathrm{Mbit} / \mathrm{s}$

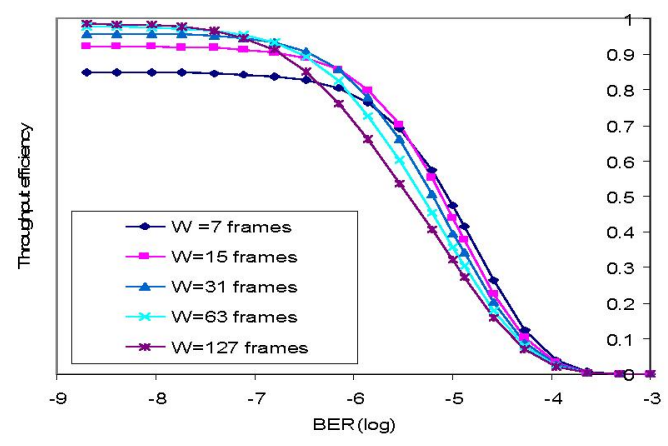

Figure 4. Throughput efficiency versus BER for BWR ARQ scheme with $t_{t a}=0.1 \mathrm{~ms}, L=2 \mathrm{~KB}$ and $C=100 \mathrm{Mbit} / \mathrm{s}$.

frames results in the same throughput (98\%) as using the GBN scheme with $W=127$ for low bit error rate and provides throughput in usable range for a wide range of bit error rate including high BER. The improvement of the throughput using the BWR ARQ scheme Compared to the existing GBN scheme with $W=127$ is significant over a wide range of bit error rate (from $10^{-7}$ to $10^{-4}$ ). It is also shown that the proposed scheme with $W=127$ provides almost the same robustness as using GBN scheme with $W=7$ but at the same time it provides almost $15 \%$ higher throughput than the GBN scheme for lower BERs. This improvement is achieved because in BWR ARQ scheme, all the error free but out of sequence frames following the first erroneous frame are stored in the buffer and most of them are not retransmitted.

The effect of increasing window size on the throughput efficiency of proposed BWR ARQ scheme for $100 \mathrm{Mbit} / \mathrm{s}$ link with $t_{t a}=0.1 \mathrm{~ms}$ and $L=2 \mathrm{~KB}$ is shown in Fig. 4. Window size increase results in some improvement in throughput efficiency for low BERs but significant decrease for high BERs. Excellent throughput efficiency at low BERs can be achieved for window size $W=127$ frames but the throughput is very much vulnerable to the high BER. The figure also depicts that window size $W=31$ frames can achieve almost the same throughput at low BERs and the performance degradation due to high BER is considerably less. So, for our proposed scheme, we will consider the window size as 31 as it provides an excellent performance over a wide range of BERs compared to the throughput using other window sizes. This also adds a small sized buffer both at the primary and the secondary station. Henceforth we will only consider the window size $W=31$ frames to analyze the performance our proposed scheme.

Fig. 5 shows throughput efficiency of BWR ARQ scheme over $100 \mathrm{Mbit} / \mathrm{s}$ links using $W=31$ for different minimum turnaround time $\left(t_{t a}\right)$. The figure shows that turn around time has significant effect on the throughput efficiency at low BERs. There is an immense improvement in throughput efficiency for decrease in $t_{t a}$ from $1.0 \mathrm{~ms}$ to $0.1 \mathrm{~ms}$ though the improvement is not at the same ratio for further decrease in $t_{t a}$ from $0.1 \mathrm{~ms}$ to $0.01 \mathrm{~ms}$. The figure also reveals that the throughput performance is almost the same if $t_{t a}$ is decreased from $0.01 \mathrm{~ms}$ to $0.00 \mathrm{lms}$. Therefore, very small $t_{t a}(0.01 \mathrm{~ms})$ is not necessary. 


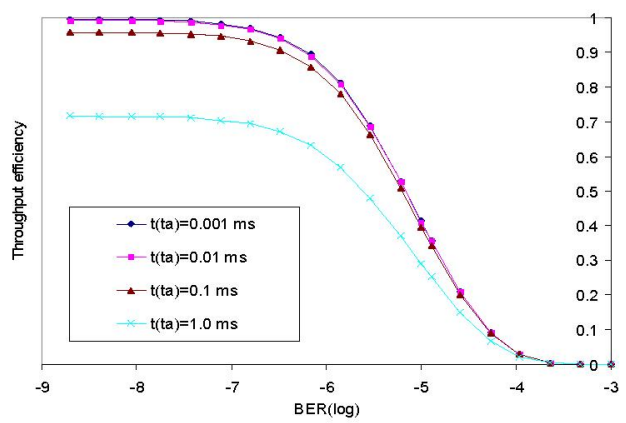

Figure 5. Throughput efficiency versus BER for BWR with $W=31$ frames, $L=2 \mathrm{~KB}$ and $C=100 \mathrm{Mbit} / \mathrm{s}$.

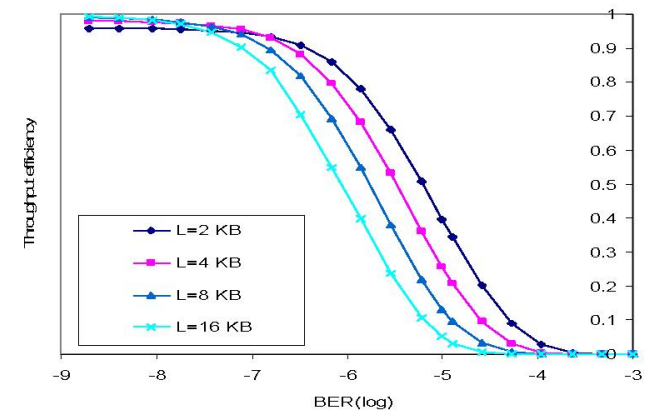

Figure 6. Throughput efficiency versus BER for BWR with $W=31$, $t_{t a}=0.1 \mathrm{~ms}$ and different frame data length $(L)$.

The effect of frame data length $(L)$ on the throughput efficiency at different BERs is shown in Fig. 6. The figure depicts that the throughput efficiency can be increased to the level of $99 \%$ using $L=16 \mathrm{~KB}$ at low BER. As we decrease $L$ by half of the previous size $(L=8 \mathrm{~KB})$, the throughput also decreases to a narrow extent but the robustness to high BERs increases. For the data length $L=2 \mathrm{~KB}$ the throughput is at the satisfactory level of $95 \%$ for very low BER. But for this data length the throughput remains almost over $80 \%$ even at high $\operatorname{BER}\left(10^{-6}\right)$.

\section{COnClusions}

In this paper, we examined the performance of a new automatic repeat request (ARQ) scheme, Block Window Retransmission (BWR) ARQ scheme, to alternate the existing go-back- $N$ (GBN) ARQ scheme for next generation high speed IrDA links. Simulation results show that throughput is significantly improved by employing the proposed BWR ARQ scheme for $100 \mathrm{Mbit} / \mathrm{s}$ IrDA links even at high bit error rates. The effects of window size, minimum turn around time and data frame length on the performance of the proposed ARQ scheme are also explained. Finally the proposed scheme saves some energy consumption for the transmitter by reducing the number of correctly received out of sequence frames to be retransmitted.

\section{REFERENCES}

[1] D. Heatley, D. Wisely, I. Neild and P. Cochrane, "Optical wireless: the story so far," IEEE communications magazine, Vol. 36, No.12, pp.72$82,1998$.

[2] Williams S., "IrDA: Past, Present and Future", IEEE Personal Communications, vol. 7, No.1, pp11-19, Feb. 2000.

[3] IrDA, Serial Infrared Link Access Protocol (IrLAP), Version 1.1, Infrared Data Association, 1996.

[4] V. Vitsas and A. C. Boucouvalas, "Optimization of IrDA IrLAP Link Access Protocol," IEEE Trans. Wireless Commun., vol.2, no.5, pp.926938, Sep.2003.

[5] T. Ozugur, M. Naghshineh and P. Kermani, "Comparison of go-back-N and selective reject ARQ modes of HDLC over half-duplex and fullduplex IR links and the effects of window size and processor speed in utilization," IEEE International Symposium on Personal, Indoor and Mobile Radio Communications, vol.2, pp.708-712, Sept.1998.

[6] A. C. Boucouvalas and V. Vitsas, "100Mb/s IrDA Protocol Performance Evalution," Proc. IASTED International Conference on Wireless and Optical Communications, pp.49-57, June, 2001.

[7] A.C. Boucouvalas and V. Vitsas: 'Optimum window and frame size for IrDA links' Electronies Letters 1st February 2001 Vol. 37, No. 3 pp.194-196.

[8] P. Barker and A. C. Boucouvalas, "Performance Modeling of the IrDA Protocol for Infrared Wireless Communications," IEEE Commun. Mag., vol. 36, no.12, pp.113-117, Dec. 1998.

[9] OPNET $^{\mathrm{TM}}$ modeller, MIL3 Inc., 3400 International Drive NW, Washington DC 20008, USA. 